\title{
An expert opinion of the Heart Failure Association of the Polish Cardiac Society on the 2021 European Society of Cardiology guidelines for the diagnosis and treatment of acute and chronic heart failure: Heart failure guidelines from a national perspective
}

\author{
Małgorzata Lelonek ${ }^{1}$, Marcin Grabowski², Jarosław D Kasprzak³, Przemysław Leszek4, Jadwiga Nessler ${ }^{5,6}$, \\ Agnieszka Pawlak ${ }^{7,8}$, Piotr Rozentryt ${ }^{9}, 10$, Ewa Straburzynska-Migaj ${ }^{11}$, Paweł Rubiś ${ }^{12,13}$
}

Reviewers: Zbigniew Gąsior ${ }^{14}$, Jarosław Kaźmierczak ${ }^{15}$

\author{
'Department of Noninvasive Cardiology, Medical University of Lodz, Łódź, Poland \\ ${ }^{2} 1^{\text {st }}$ Department of Cardiology, Medical University of Warsaw, Warszawa, Poland \\ ${ }^{3} 1^{\text {st }}$ Department of Cardiology, Medical University of Lodz, Bieganski Hospital, Łódź, Poland \\ ${ }^{4}$ Department of Heart Failure and Transplantology, National Institute of Cardiology, Warszawa, Poland \\ 5Department of Coronary Disease and Heart Failure, John Paul II Hospital, Kraków, Poland \\ ${ }^{6}$ Department of Coronary Disease and Heart Failure, Institute of Cardiology, Jagiellonian University Medical College, Kraków, Poland \\ ${ }^{7}$ Department of Invasive Cardiology, Central Clinical Hospital of the Ministry of Interior and Administration, Warszawa, Poland \\ ${ }^{8}$ Mossakowski Medical Research Center, Polish Academy of Sciences, Warszawa, Poland \\ 'Department of Toxicology and Health Protection, Faculty of Health Sciences in Bytom, Medical University of Silesia in Katowice, Bytom, Poland

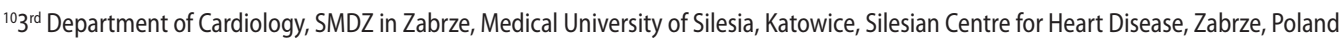 \\ ${ }^{11} 1^{\text {st }}$ Department of Cardiology, University of Medical Sciences in Poznan, Heliodor Święcicki University Hospital, Poznań, Poland \\ ${ }^{12}$ Department of Cardiac and Vascular Diseases, Institute of Cardiology, Jagiellonian University Collegium Medicum, Krakow, Poland \\ ${ }^{13}$ John Paul II Hospital, Kraków, Poland \\ ${ }^{14} \mathrm{Chair}$ and Clinic of Cardiology, Faculty of Health Sciences in Katowice, Medical University of Silesia, Katowice, Poland \\ ${ }^{15}$ Clinic of Cardiology, Faculty of Medicine and Dentistry, Pomeranian Medical University, Szczecin, Poland
}

Correspondence to:

Prof. Małgorzata Lelonek, MD,

PhD, FESC, FHFA,

Department of Noninvasive

Cardiology,

Medical University of Lodz

Żeromskiego 113, 90-549 Łódź,

Poland,

phone: +484263937 93,

e-mail:

malgorzata.lelonek@umed.lodz.pl

Copyright by the Polish Cardiac

Society, 2022

DOI: 10.33963/KP.a2022.0021

Received:

January 9, 2022

Accepted:

January 9, 2022

Early publication date:

January 25,2022

\section{A B S TR A C T}

The Polish expert opinion of the Heart Failure Association of the Polish Cardiac Society on the 2021 European Society of Cardiology (ESC) guidelines for the diagnosis and treatment of heart failure points to differences in many aspects related to heart failure in Poland compared with other European countries. These differences include population issues, epidemiology, diagnostic and treatment options, or the organization of healthcare. This expert opinion also includes a review of new results of clinical trials completed after the publication of the ESC guidelines.

Key words: heart failure, diagnosis and therapy, multidisciplinary approach, guidelines

\section{INTRODUCTION}

A panel of experts from the Heart Failure Association of the Polish Cardiac Society read with great interest the long-awaited 2021 European Society of Cardiology (ESC) guidelines for the diagnosis and treatment of acute and chronic heart failure (HF) [1]. The current update brings into clinical practice numerous novel concepts and diagnostic and therapeutic pathways. Naturally, all the guidelines are prepared in the best interests of patients who are truly the subject of the document. On the other hand, every ESC country member has their own unique features, including population structure, geography, gross domestic product, etc. that ultimately is translated into unique healthcare policies. Bearing this in mind, the expert panel 
critically revised this document, pointing out its novelty, as well as the reality of Polish patients and physicans who operate within our current healthcare system.

\section{COMMENT ON HF DEFINITION, EPIDEMIOLOGY, AND PROGNOSIS (CHAPTER 3)}

A universal definition of HF was published earlier this year. Linking structural and/or functional abnormalities of the heart with typical symptoms remains the mainstay of clinical diagnosis. A unique shift in the classification of $\mathrm{HF}$ is the renaming of patients who present with their left ventricular ejection fraction (EF) between $41 \%$ and $49 \%$. These patients used to be described as having HF with midrange; now the term that is proposed is mildly reduced EF (notably, the abbreviation does not change - HFmrEF). This emphasizes recently shown similarities between HF with reduced $\mathrm{EF}$ (HFrEF) and HFmrEF.

Key information related to the epidemiology and prognosis in HF had no major changes; however, a specific point of interest is how the conditions in Poland compare to general statements found in the guidelines. Like other countries, improvements in therapies are counterbalanced by the increase in HF prevalence due to the aging of the general population. According to the recent national report, in 2018, the prevalence of the disease in the Polish population was 1240000 subjects, with an increasing yearly incidence, followed by rising hospitalizations [2]. Notably, Poland is the Organization for Economic Co-operation and Development (OECD) country with the highest rate of HF hospitalizations at 594 for 100000 citizens. This is accompanied by the rise in healthcare system costs related to the imbalanced HF rate in our country. Moreover, the number of deaths hit the record of 142000 in the HF population (with $\mathrm{HF}$ as the direct cause in $41000-9.8 \%$ of all deaths) in year 2018. Notably, more recent data encompassing pandemic period is not available as yet.

\section{COMMENT ON HF DIAGNOSIS (CHAPTER 4)}

The key diagnostic flowchart does not conceptually differ from the previous edition and in Poland's clinical practice, echocardiography probably still often precedes natriuretic peptides; both tests allow for the rejection of the HF hypothesis when normal (94\%-98\% negative predictive value for natriuretic peptide tests). The changes in diagnostic recommendations include an increased indication class for coronary computed tomography angiography with less emphasis on invasive coronary angiography. Right heart catheterization is recommended before transplant evaluation. This can also be included in the workup of HF with preserved $\mathrm{EF}$ (HFpEF), as well as with the less common HF etiologies, such as constrictive pericarditis, restrictive cardiomyopathy, congenital heart disease or high cardiac output conditions if clinically suspected. Regarding cardiac imaging in healthcare Polish environments, there is extensive expertise in echocardiographic stress testing but a remarkable shortage of dedicated cardiac magnetic resonance (CMR) services, indispensable for the diagnosis of less common myocardial diseases, including amyloidosis, and infiltrative and inflammatory conditions.

\section{COMMENT ON HFrEF (CHAPTER 5)}

The current guidelines emphasize that pharmacotherapy is the cornerstone of HFrEF treatment, advising that it be initiated before considering all other interventions, or even used simultaneously [1]. The field of pharmacotherapy is not merely introducing novel groups of drugs but witnessing a paradigm shift in the treatment of patients with HFrEF [1], i.e. the turn towards an individual approach to treatment depending on patients' clinical profiles [3]. The newest expert proposal for the treatment of HFrEF assumes that the 4 groups of recommended drugs should be, if possible, initiated simultaneously or in stages, depending on patients' clinical profiles, in a period not exceeding 4 weeks. After that time, the dosing ought to be optimized [4]. The two algorithms presented in the ESC guidelines are useful in selecting the appropriate treatment options for patients with HFrEF - a very valuable addition, in our view. The first one presents a treatment strategy to reduce mortality, including the indications for using first-class recommendation drugs and devices, in patients with $\mathrm{HFrEF}$ while also taking into account the etiology of HF [1]. The second algorithm is a central illustration providing a phenotypic overview of the treatment of HFrEF, including its etiology, patient clinical characteristics, the stage of the disease, and comorbidities. It also emphasizes the need for cardiac rehabilitation for all patients and for enrolling them into multidisciplinary care programs [1].

Presently, four groups of drugs are considered to be pivotal (class I) in the treatment of patients with HFrEF. In addition to the previously recommended angiotensin-converting enzyme inhibitors (ACE-I) or angiotensin receptor-neprilysin inhibitor (ARNI), beta-blockers, and mineralocorticoid-receptor antagonists (MRA), we can see that a new group of drugs has been added: the sodium-glucose co-transporter type 2 inhibitors (SGLT2i), commonly known as flozins.

In addition to the above-mentioned disease-modifying drugs, diuretics are at the top of the algorithm. The effect of this class of drugs on cardiovascular morbidity and mortality has not been studied in randomized controlled trials. However, it should be remembered that every major trial of disease-modifying therapies in HFrEF has been conducted on top of loop diuretic therapy. The current ESC guidelines emphasize that patients should be trained to self-titrate diuretic doses based on self-monitoring for symptoms or signs of congestion and daily body weight measurements [1].

In HFrEF, it took a considerable length of time to replace ACE-I with a more effective class of medication - sacubitril/valsartan, the only representative of the ARNI group. Several studies published in the meantime indicate that 
ARNI can be considered a first-line therapy instead of ACE-I. Therefore, the indications for the use of ARNI have been significantly expanded, especially in acute decompensated HFrEF after hemodynamic stabilization [5-7]. In this population, ARNI is safe, and it reduced cardiovascular mortality and HF hospitalizations by $42 \%$ when compared to enalapril [7]. The clinical benefit was greater in de novo patients who were previously untreated and hospitalized due to HF. Therefore, the initiation of sacubitril/valsartan treatment may be considered in ACE-I naïve patients with HFrEF before discharge (recommendation class IIb, evidence level B). Considering all the available evidence proving the effectiveness of ARNI, in our opinion, ACE-I should be replaced with $A R N I$ in these patients whenever it is possible, and initiation of therapy with this drug should even be considered in de novo patients. However, in Poland, unlike in almost all other countries in the EU, sacubitril/valsartan is still not reimbursed. Fortunately, over the recent years, the producer of sacubitril/valsartan significantly decreased the cost of this therapy for individual HFrEF patients. As a result, this therapy became available for an increasing number of patients with HFrEF, but still not for all of them. Angiotensin II receptor blockers (ARBs) are currently on more far position in guidelines (no benefit in reducing mortality) and recommended for patients who are intolerant to ACEls or ARNIs due to serious adverse events [1].

What undoubtedly constitutes a breakthrough therapy in HFrEF is the arrival of SGLT2i - dapagliflozin and empagliflozin. Adding SGLT2i to standard guideline-based therapy significantly reduces the risk of cardiovascular death and worsening of HF in a short time after treatment initiation (within 1 month). Due to their unique mechanism of action, these drugs can be used at different stages of the metabolic-cardio-renal continuum. Furthermore, these drugs are particularly straightforward to use (1 tablet taken once daily without any need for dose modification) and are well-tolerated and safe (without the requirement for close monitoring of electrolytes or renal parameters). Currently, both dapagliflozin and empagliflozin are recommended for all patients with HFrEF regardless of whether they have type 2 diabetes or not [1]. In some patients with a de novo diagnosis of HFrEF, and especially patients with a profile including low arterial pressure and impaired renal function - flozins may be, next to beta-blockers, the first drug of choice, which will then enable the use of other recommended I class medications.

The new 2021 ESC guidelines also included vericiguat the first-in-class guanylate cyclase receptor stimulator which was assessed in patients with HFrEF and a recent decompensation of HF [1]. Unfortunately, vericiguat is not currently available in Poland.

\section{COMMENTS ON CARDIAC RHYTHM MANAGEMENT IN HFrEF (CHAPTER 6)}

According to the new document, the indication for cardioverter-defibrillator implantation (ICD) in secondary pre- vention remains unchanged; it is a class I recommendation if there is an expected $>1$-year survival in good functional status, with no reversible causes of cardiac arrest and when there has been no early ventricular arrhythmia ( $<48$ hours) following the onset of acute myocardial infarction. Mainly, as a consequence of the DANISH study, the new document makes distinctions in the strength of its recommendations for the ICD in primary prevention ( $\mathrm{EF} \leq 35 \%$ ) based on $\mathrm{HF}$ etiology: ICD has become class I for ischemic HF and class Ila for non-ischemic etiology. The guidelines highlight the need to re-evaluate the indications for continuing ICD therapy by an experienced cardiologist at the time of generator elective replacement (class Ila). They also note no indications for ICD (class III) in NYHA class IV patients with severe recurrent symptoms despite pharmacotherapy, unless they are candidates for cardiac resynchronization therapy (CRT), a left ventricular assist device (LVAD), or heart transplantation (HTX). The guidelines also mention a wearable cardioverted-defibrillator (WCD) as a therapy to prevent sudden death in a shorter period or as a bridge to the implantation of an ICD (class IIb). Although WCD is becoming increasingly popular in a subset of patients in Poland, there is still room for improvement of WCD availability, including unlimited reimbursement for those in need. The indications for a subcutaneous cardioverter-defibrillator are comparable to those for transvenous devices with a preference for patients with expected long-term survival and at increased risk of infectious and vascular complications. Subcutaneous devices are not recommended in patients with indications for cardiac pacing, antiarrhythmic pacing, or CRT. The main recommendations for CRT are a wide QRS ( $\geq 150 \mathrm{~ms}$ ) with a morphology of the left bundle branch block type (LBBB) (class I). The class I recommendation for CRT to reduce mortality also applies, irrespective of the NYHA class and the QRS width, in patients with indicated ventricular pacing due to a high-grade AV block, including patients with atrial fibrillation. In the new guidelines, on the one hand, the recommendation class was reduced (from I to lla) for CRT in symptomatic patients with sinus rhythm, EF $\leq 35 \%$, and the QRS width 130-149 ms with LBBB morphology. On the other hand, the recommendation was increased (from Ilb to Ila) for upgrading to CRT in patients with a previously implanted ICD or conventional pacemaker, who have a high percentage of ventricular pacing.

The guidelines also mention other devices currently under investigation, such as systems that modify the activity of the autonomic nervous system or cardiac contractility modulation (CCM). CCM was evaluated in NYHA III-IV patients with EF $25 \%-45 \%$ and the QRS width $<130 \mathrm{~ms}$, which may reduce symptoms of exercise intolerance and improve the quality of life. The guidelines do not specify any differences in indications for CRT-P (CRT-pacemaker) alone or CRT with the cardioverter-defibrillator option (CRT-D). According to the simultaneously published ESC guidelines on cardiac pacing and resynchronization, CRT-P may be preferred over CRT-D in patients with non-ischemic 
cardiomyopathy, with shorter expected survival, multiple comorbidities, impaired renal function, or patient preference [8]. Summarizing the guidelines for cardiac electrotherapy, from the perspective of our practice, it is clear that the guidelines place an emphasis on the patient obtaining full, optimal pharmacotherapy (including sacubitril-valsartan and SGLT2i) used over a sufficiently long period before qualifying for electrotherapy.

In the treatment of coexisting atrial fibrillation, the position of non-vitamin $\mathrm{K}$ antagonist oral anticoagulants (NOACs) over vitamin $\mathrm{K}$ antagonists (VKAs), and the role of catheter ablation (for heart rate control in symptomatic patients despite optimal pharmacotherapy) have been strengthened by being made class I and Ila recommendations, respectively. It is important to underscore the increased role of NOACs here, which should be preferred over VKAs (class I), with the limitation of their use in patients with artificial mechanical valves, and moderate/severe mitral stenosis. In the cases where there is a clear association between paroxysmal or persistent atrial fibrillation and worsening of HF symptoms, catheter ablation should be considered for the prevention or treatment of atrial fibrillation. The role of beta-blockers has been reduced (downgraded from class I to Ila). In patients with atrial fibrillation and poor ventricular rate control despite pharmacotherapy, simultaneous AV node ablation and CRT implantation can be considered.

\section{COMMENT ON HFMrEF (CHAPTER 7)}

For the first time in the ESC guidelines for HF, therapy dedicated to HFmrEF has been described. There have been no specific randomized controlled trials in HFmrEF; however, some trials in $\mathrm{HFpEF}$ included patients with $\mathrm{EF}>40 \%$, and from the sub-analyses, some data have emerged. Four groups of pharmacological therapies are described in the guidelines with class IIb and C levels for HFmrEF: ACE-Is, ARBs, $\beta$-blockers, MRAs, and ARNIs. However, sacubitril/valsartan (ARNI) is only approved to treat patients in a broad spectrum of HF (both HFrEF and HFpEF) by the FDA but not in Europe by the EMA (in Europe only in HFrEF).

\section{COMMENT ON HF WITH PRESERVED EF (HFpEF) (CHAPTER 8)}

The identification of patients with HFpEF remains challenging. In our country, no data exists on the number of patients with HFpEF. The diagnostic approach to HFpEF includes natriuretic peptides; however, general practitioners in Poland have no means of measuring this biomarker. So, the diagnosis of HFpEF is usually made by cardiologists or at the hospital during HF hospitalization. In the 2021 ESC guidelines, no specific treatment for HFpEF is given; nonetheless, ESC guidelines still recommend the screening and treatment of risk factors and comorbidities. Despite the positive results of EMPEROR-Preserved (Empagliflozin Outcome Trial in Patients With Chronic Heart Failure With Preserved Ejection Fraction), there are no recommenda- tions in the present guidelines regarding empagliflozin for the treatment of HFpEF due to the parallel timelines of the publication of this document and the EMPEROR-Preserved results [9].

\section{COMMENT ON MULTIDISCIPLINARY TEAM MANAGEMENT FOR THE PREVENTION AND TREATMENT OF CHRONIC HF (CHAPTER 9)}

For the first time, a special chapter is dedicated to patient education, self-care, and lifestyle advice. New recommendations are described for a self-management approach, and either home-based and/or clinic-based programs to reduce the risk of HF hospitalization and mortality (class I). Unfortunately, in our country, HF management programs are still unavailable.

\section{COMMENT ON ADVANCED HEART FAILURE (CHAPTER 10)}

In the new 2021 guidelines, also for the first time, there is a separate chapter centered on advanced HF, in which its epidemiology, diagnostics, prognosis, and management are discussed. With the recent progress in HF treatment, the prolongation of survival among HF patients, the decrease in the risk of sudden cardiac death, the number of advanced HF patients is growing. It is estimated that $1 \%-10 \%$ of patients with $\mathrm{HF}$ are at an advanced stage of $\mathrm{HF}$, and this highlights the importance of the proper and early diagnosis of advanced $\mathrm{HF}$ and the referral of these patients to tertiary centers. The updated HFA-ESC 2018 definition of advanced $\mathrm{HF}$ is underlined. What is new in these guidelines is that starting the use of continuous inotropes and/or vasopressors may be considered a bridge to mechanical circulatory support (MCS) or HTX.

The INTERMACS (Interagency Registry for Mechanically Assisted Circulatory Support) classification, which characterizes advanced HF patients under consideration for MCS implantation, an elegant algorithm for the treatment of patients with advanced HF is clearly described, and crucially, applied. The current guidelines stress the proper referral of suitable candidates at the right time for LVAD implantation. Unfortunately, MCS is still underused in Poland, and awareness-raising about this treatment type should be urgently initiated among Polish cardiologists. Encouragingly, new generations of these devices offer an $80 \%$ chance of 1-year survival and a $70 \%$ chance of 2 -year survival.

However, the gold standard of treatment for advanced $\mathrm{HF}$ remains $\mathrm{HTX}$, which is linked to an approx. $90 \%$ rate of 1-year survival, and 12.5 years of median survival. In Poland, there are still too few HTX procedures. In principle, indications and contraindications for HTX have not changed for several years. Advanced age (>65 years) is no longer an absolute contraindication, and in some centers, patients of up to 70 years are accepted while taking into account their biological age.

The decision pathway to HTX or LVAD is a complex process and is quite individual for each patient. What must 
be considered is not just indications but also a long list of contraindications and limitations. In addition, the care of the patients following HTX and LVAD is of utmost importance, and it requires good cooperation and motivation from patients and their relatives and a readiness on the part of the center to assist the patients round the clock. While a patient post-HTX has a healthy heart, a patient with an LVAD is still an HF patient, taking HF medications and at risk of decompensation, e.g. worsening of the right ventricle (not mechanically assisted). In Poland, in the period 2019-2020, 290 HTXs were performed, and since then, up to November 2021, more than 160 procedures were carried out.

End-of-life care and key components of palliative care in advanced HF are also described. The management of advanced $\mathrm{HF}$ is based mainly on expert experience and opinions. Urgent research dedicated to advanced HF is required, necessitating the development of novel inotropes and myotropes, as well as the development of new treatment algorithms which could have a positive impact on survival, quality of life, and hospitalization rates.

\section{COMMENT ON ACUTE HF (CHAPTER 11)}

In a new section, the authors clearly distinguish individual phenotypic forms of acute HF (AHF) while also combining phenotypes with treatment tactics, all presented in a very straightforward scheme. So, although there has been no breakthrough in the drugs available for AHF treatment, the tactics have been clarified, which makes this part helpful for the physician treating a patient with AHF.

The authors drew attention to the fact that clinical severity and patient outcomes are determined by a complex interplay between precipitants, the underlying cardiac substrate, and the patients' comorbidities. The management of AHF patients is presented with due consideration of all these factors. Among the initial AHF diagnostic tests, apart from the classical ones, new recommendations for tests have appeared:

- LUS (lung ultrasound) for congestion assessment and monitoring;

- Iron status (transferrin, ferritin) for iron deficiency assessment;

- Lactate for perfusion status assessment.

Based mainly on the presence of signs of congestion and/or peripheral hypoperfusion, AHF clinical presentations were described as 4 major clinical phenotypes:

- Acutely decompensated heart failure - distinct from pulmonary edema - this phenotype is the most common form of AHF (50\%-70\%);

- Acute pulmonary edema;

- RV failure;

- Cardiogenic shock.

For the in-hospital management of AHF, a new acronym was established - CHAMPIT — extended with I for infection and T for tamponade. In pre-discharge and post-discharge management, for the first time, the recommendations for the management of patients after HF hospitalization are described with careful evaluation of any signs of congestion. The administration before the discharge of evidence-based oral medical treatment and the treatment of comorbidities (with iron deficiency making its first appearance in the guidelines) are presented as well. There is a recommendation made to schedule one follow-up visit within 1 to 2 weeks after discharge, which in Poland, but perhaps in other countries as well, is hard to execute. The guidelines do not specify who exactly should make this follow-up visit - general practitioners, cardiologists, or HF nurses. To date, there are no HF nurses in Poland; however, a newly-developed educational platform now exists for the certification of HF nurses (www.edu.slabeserce.pl).

The selected treatment recommendations used for AHF were refined:

- Diuretics (class I/level of evidence C) treatment should be started intravenously and, based on the diuretic response, related to spot urine sodium content and/or the hourly urine output measurement after 2 or 6 hours. If the diuretic response is insufficient, the loop diuretic i.v. dose should be doubled, with a later assessment of diuretic response. Concomitant administration with diuretics acting at different sites, namely thiazides or acetazolamide, may be considered (Ila B). In Poland, the measurement of urine sodium content is rarely performed despite its low cost;

- Vasodilators may be considered intravenously as initial therapy in patients with systolic blood pressure $>110 \mathrm{~mm} \mathrm{Hg}$ with a lower class/level Ilb/B than in the 2016 guidelines.

- From Inotropes (class/level IIb/C), Levosimendan (non-adrenergic mechanisms) still holds a position that is too low, in our opinion. Even though the current guidelines recommend Levosimendan over dobutamine for patients on $\beta$-blockers, Polish physicians still underuse this drug, apart from centers specialized for $\mathrm{HF}$, where the situation is more satisfactory [10];

- Opiates (class/level III/C), the routine use of opiates in AHF is now not recommended, although they may be considered in selected patients, particularly in the case of severe/intractable pain or anxiety, or in the setting of palliation.

It is worth noting that the results of the EMPULSE study (Empagliflozin Compared to Placebo Initiated in Patients Hospitalized for Acute Heart Failure Who Have Been Stabilized) were presented on November 14, 2021, at the American Heart Association Congress. The benefits of empagliflozin initiation in patients hospitalized for AHF resulted $36 \%$ more likely (stratified win ratio, 1.36; $95 \%$ $\mathrm{Cl}, 1.09-1.68 ; P=0.0054)$ to experience a clinical benefit, including reduced all-cause mortality, HF events, and an improvement in HF symptoms versus placebo during a 90day follow-up, regardless of EF and diabetes status. 


\section{COMMENT ON CARDIOVASCULAR COMORBIDITIES (CHAPTER 12)}

The management of cardiovascular comorbidities remains a key component of proper HF care. In HFrEF patients with concomitant chronic coronary syndromes, myocardial revascularization should be considered when angina persists despite the use of beta-blockers supplemented by other antianginal drugs. Medical therapy should also facilitate the control of hypertension, with at least standard treatment targets; mild asymptomatic hypotension may be acceptable in HFrEF if caused by target doses of prognosis-related drugs. All ACE-I, HF-approved beta-blockers, and diuretics are advisable as pillars of blood pressure control, whereas other classes remain a second choice. Correcting advanced valve disease contributing to or causing HF is beyond debate in patients with limited comorbidities, and percutaneous techniques (TAVI, MitraClip) play an increasingly important role in these high-risk patients. We were surprised to read a caution against beta-blockers in aortic regurgitation - there is neither clinical nor evidence-based data to support such a concern. In secondary mitral regurgitation (MR), the readership should pay much attention to the current concept of the actively defined patient subgroup with disproportional MR [11]. Patients with a high regurgitant volume-to-LV end-diastolic volume ratio and with an absence of any significant right-sided disease component or terminal LV disease are optimal candidates for targeting MR with Mitraclip (COAPT-like population). Wider access to percutaneous mitral and tricuspid interventions is necessary but poses a critical challenge for the Polish healthcare system due to the restrictive policy of the National Health Fund.

\section{COMMENT ON NON-CARDIOVASCULAR COMORBIDITIES (CHAPTER 13)}

In real clinical day-to-day experience, HF is rarely the only clinical problem. According to different registries, each HF patient suffers from 3 to 5 other chronic diseases, usually regarded as comorbidities. In the recent ESC guidelines, this issue has attracted a great deal of attention. Here, we would like to provide a subjective commentary. All chronic pathologies accompanying HF can be grouped into different categories. The authors of the guidelines divided them into the categories of cardiovascular (chapter 12), and non-cardiovascular (chapter 13). All of the comorbidities mentioned in the guidelines may either facilitate the development of de novo HF or may precipitate acute decompensations. However, their coexistence with HF may vary over different timescales. Given that chronic disease may either precede HF (working as an etiologic or co-etiologic factor, i.e. diabetes, chronic kidney disease, sarcopenia) or develop during its course, any knowledge obtained concerning the chronology of the diseases may be of importance to clinicians. By design, based on the guidelines' development, each chronic disease is described as a sole companion of HF. However, in real life, certain comorbidities aggregate in $\mathrm{HF}$, based mostly on the pathophysiology shared with the HF condition. Such specific clusters of chronic diseases produce characteristic clinical phenotypes and - critically - may differ in terms of prognosis.

Chronic obstructive pulmonary disease (COPD) and HF share numerous risk factors and their clinical presentation may be similar. Careful examination in the congestion-free phase is a key issue because clinical and even spirometry testing may show significant reductions in bronchial flow in incompletely decongested patients. The difference between forced expiratory volume in one second expressed as $\%\left(\mathrm{FEV}_{1} \%\right)$ before and after the resolution of congestion may be as high as 15\%-20\% [12]. Current guidelines suggest that the use of inhaled broncho-dilators in AHF presenting with a reduction of $\mathrm{FEV}_{1} \%$ may be beneficial. The basis for this suggestion stems from a single study in which patients with COPD and documented hyperinflation received indacaterol/glycopyrronium or placebo, in a cross-over design. The study showed a slight decrease in end-diastolic volume in patients receiving active treatment, which was interpreted as an improvement in cardiac function [13]. This finding requires special comment. It is worth noting that the patients in this study were free from $\mathrm{HF}$, and one should be extremely cautious about expecting similar effects in HF patients, especially in those with extremely dilated ventricles. Such patients may not be able to increase their contractility force in response to further dilation as the gain of Starling's law may be exhausted, leading to the opposite effect and the reduction of stroke volume.

Worsening of kidney function arising from chronic and acute kidney disease in HF are both important comorbidities. There is no doubt that chronic kidney disease aggravates HF course and negatively impacts prognosis. However, deteriorating kidney function may be associated with both worse and better prognoses. The key issue is the clinical context because poor kidney function in patients who are responding positively to treatment (for example by decreasing natriuretic peptide levels) is associated with better outcomes, while the opposite is true when the clinical response is absent, or merely marginal.

New in the current guidelines is the extensive discussion on abnormalities such as hyponatremia, hypochloremia, metabolic alkalosis, and hyperkalemia, as important comorbidities. Apart from their possible iatrogenic genesis, we believe that they reflect other aspects of kidney dysfunction, namely the exhaustion of kidney homeostatic regulatory potential in each patient-specific pathophysiological and HF treatment environment. Their occurrence should prompt careful examination in an effort to tailor therapy to the specific clinical situation.

Also for the first time, there are recommendations for the management of patients with HF and iron deficiency (ID), which is related to the results of the AFFIRM-HF study [14]. In HF, it is now recommended that all patients undergo periodic screening for ID and anemia (class I), and intravenous iron supplementation with ferric car- 
boxymaltose in symptomatic patients, either recently hospitalized for HF with $\mathrm{EF} \leq 50 \%$ or with $\mathrm{EF}<45 \%$ (class Ila). Unfortunately, ID is still underdiagnosed in Poland and thus, there is a need to increase awareness of this deficiency among physicians.

\section{COMMENT ON CARDIOMYOPATHIES AND MYOCARDITIS (CHAPTER 14)}

Uniquely, and for the first time, in the current HF guidelines, there is a separate section entitled "Special Conditions" (section 14), which includes topics devoted exclusively to pregnancy, cardiomyopathies, left ventricular non-compaction, atrial disease, myocarditis, amyloidosis, iron overload cardiomyopathy, and adult congenital heart disease [1]. This inclusion highlights the rapid development of this critically important field within the whole HF spectrum. All the information contained in this section is beyond the scope of this concise commentary; nonetheless, the most essential items and novel features are briefly presented below:

- New guidelines underline various direct causes of cardiomyopathies, and conditions classified as disease modifiers. The guidelines urge that specific causes of cardiomyopathies should be sought out;

- For each main cardiomyopathy type, there is a detailed section on genetic testing with a minimal set of genes deemed mandatory. Clear recommendations are given on genetic counseling in index patients and relatives;

- Interestingly, above and beyond the well-known main cardiomyopathy types, such as dilated (DCM) and hypertrophic (HCM) cardiomyopathy, the current guidelines also introduce the novel concept of arrhythmogenic cardiomyopathy (AC) [15]. This is a much broader term than the "classic" arrhythmogenic right ventricular cardiomyopathy (ARVC), typically restricted to the RV and, instead, acknowledges that left ventricular (LV) involvement occurs in approximately one-third of cases;

- Given that beneficial LV reverse remodeling (LVRR) occurs in approximately $50 \%$ of DCM patients, the question arises as to whether to continue HF-modifying treatment in those with LVRR [16]. This issue was tackled by the authors of the TRED-HF study, where it was shown that withdrawal of treatment resulted in $\mathrm{HF}$ relapse in up to $44 \%$ of DCM patients [17];

- The guidelines spotlight a possible first-of-its-kind disease-specific treatment in HCM. The EXPLORER-HCM study reported that Mavacamten, which reduces cardiac muscle contractility by inhibiting excessive myosin-actin cross-bridge formation, improved exercise tolerance and NYHA class and reduced LV outflow tract gradient in obstructive $\mathrm{HCM}$;

- The guidelines address the controversy concerning LV non-compaction cardiomyopathy (LVNC). Although several echocardiographic and magnetic resonance criteria exist, they lack much specificity and their diagnostic accuracy is thought to be somewhat limited.
Therefore, the guidelines propose not treating LVNC as separate cardiomyopathy but rather as a rare presentation of DCM or HCM.

The document also briefly comments on iron overload cardiomyopathy (IOCM). The examination of choice to confirm myocardial iron deposition is a special magnetic resonance technique $-\mathrm{T} 2 *$.

A special sub-section (14.6) explores a hot topic in $\mathrm{HF}$ - amyloid cardiomyopathy. A rapid non-invasive diagnostic algorithm, based on the exclusion of light chain amyloidosis $(\mathrm{AL})$ and the utilization of technetium-labeled ${ }^{99 m} T c-P Y P$ or DPD or HMDP scintigraphy, has a very high specificity and yields positive predictive values for the diagnosis of transthyretin (TTR) cardiomyopathy (TTR-CM) [18]. Unfortunately, TTR amyloidosis is still underdiagnosed in Poland, and a "rising awareness" campaign should be initiated among Polish physicians. The document briefly introduces two types of TTR-CA - a wild one ( $>90 \%$ of cases) and a hereditary one - and draws attention to typical "red flags" for both TTR-CM and AL-CM, as well as a clear diagrammatic presentation of workup. Current treatment of AL-CM is based on chemotherapy and/or autologous stem-cell transplant and is normally managed by hematologists. The fruitful results of the recently published ATTR-ACT trial have paved the way for the incorporation of Tafamidis, a stabilizer of TTR tetramers into the standard treatment regime for TTR-CM and now has class Ib recommendation [19]. The dedicated drug program (with Tafamidis) for TTR-CM has been prepared and is currently under review.

The 2021 ESC guidelines in the chapter "Special conditions" also for the first time provide information on the epidemiology, etiology, diagnostics, and treatment of patients with acute myocarditis. For etiology, only three groups of causes are listed (infectious with a focus on viral agents, systemic diseases, and finally toxic), which, on balance, seems to be justified. In these groups, it is possible to determine the diagnosis and make therapeutic decisions. The diagnostic workup involves many diagnostic tests, but only three are considered methods of high or intermediate sensitivity and specificity: CMR, coronary angiography, and endomyocardial biopsy (EMB). What is new is the discussion on EMB indications is the number and sites of the biopsy specimens and the methods to be used when analyzing the biopsy material. In addition to the histological and immunohistochemical analyses, the assessment should also refer to the determination of the etiology. If viral etiology is suspected, the EMB, in addition to quantifying the viral genome (rt-PCR) for the most common cardiotropic viruses (parvovirus B19, HHV4, HHV6, enteroviruses, adenovirus and coxsackievirus, SARS-CoV-2), should also provide data on active viral replication via the evaluation of viral mRNA. A thorough analysis of the EMB specimens should lead to the right therapeutic decisions. At the present stage of knowledge, immunosuppression has been considered for the treatment of patients with chronic cardiac inflammation at EMB and with no evidence of ac- 
tive viral infection. To date, the diagnosis of myocarditis in Poland is based on CMR. It is well known that while CMR brings many advantages, it cannot establish the etiology of myocarditis. Qualitative and quantitative typing of viruses in the myocardial tissue has only been made possible thanks to research grants in individual centers in Poland. Currently, in Poland, we lack procedures financed by the National Health Fund that would allow us to conduct comprehensive diagnostic evaluation in relation to myocarditis, carry out staff training or establish professionally equipped pathology departments in referral centers. These guidelines reveal the enormous work that we must undertake in Poland to reach a more refined diagnosis of myocarditis.

\section{CONCLUSIONS}

The 2021 ESC guidelines for the diagnosis and treatment of acute and chronic HF propose and summarize the best management strategies for HF patients, including the new diagnostic and therapeutic options. However, the final decision about an individual patient is related to the capacity of the healthcare system and is taken by health professionals considering the patient's preferences. A panel of experts from the Heart Failure Association of the Polish Cardiac Society commented on the 2021 ESC guidelines based on the reality of the Polish healthcare system.

\section{Article information}

Conflict of interest: ML received lecture and consulting fees from Novartis, Servier, AstraZeneca, Boehringer Ingelheim, Bausch Health, Bayer, Gedeon Richter and were involved in clinical trials from Novartis and Behringer Ingelheim. MG received lecture and consulting fees from Novartis, Servier, AstraZeneca, Boehringer Ingelheim, Polpharma, Abbott, Boston, Biotronik, Medtronic. JDK received lecture and consulting fees from Novartis, Servier, AstraZeneca, Boehringer Ingelheim, Pfizer, Bayer, Polpharma. PL received lecture and consulting fees from Novartis, Servier, AstraZeneca, Bayer, Boehringer Ingelheim, Roche Diagnostic, Swixx Biopharma, BMS. JN received lecture and consulting fees from Novartis, Servier, AstraZeneca, Boehringer Ingelheim, Berlin Chemie. P. Rozentryt received lecture and consulting fees from Novartis, Servier, AstraZeneca, Berlin Chemie Menarini, Boehringer Ingelheim, Bayer, Pfizer, IvoPharma, Krka, Teva, Genzime, Polpharma and was involved in clinical trials sponsored by Vifor Pharma. E S-M received lecture and consulting fees from Novartis, Servier, AstraZeneca, Boehringer Ingelheim, Bayer, Pfizer, Swixx BioPharma, and was involved in clinical trials sponsored by Bayer. Other authors declare no conflict of interest.

Open access: This article is available in open access under Creative Common Attribution-Non-Commercial-No Derivatives 4.0 International (CC BY-NC-ND 4.0) license, allowing to download articles and share them with others as long as they credit the authors and the publisher, but without permission to change them in any way or use them commercially. For commercial use, please contact the journal office at kardiologiapolska@ptkardio.pl.

\section{REFERENCES}

1. McDonagh TA, Metra M, Adamo M, et al. 2021 ESC Guidelines for the diagnosis and treatment of acute and chronic heart failure. Eur Heart J. 2021; 42(36): 3599-3726, doi: 10.1093/eurheartj/ehab368, indexed in Pubmed: 34447992.

2. Heart failure in Poland Reality, costs, suggestions for improving the situation [article in Polish]. Kałużna-Oleksy M. (ed). 2020. Available online:
https://marfan.org.pl/wp-content/uploads/2021/01/Niewydolnos\%CC\%81c\%CC\%81-RAPORT-A4-2021-NET.pdf. (Access: January 25, 2022).

3. Rosano GMC, Moura B, Metra $M$, et al. Patient profiling in heart failure for tailoring medical therapy. A consensus document of the Heart Failure Association of the European Society of Cardiology. Eur J Heart Fail. 2021; 23(6): 872-881, doi: 10.1002/ejhf.2206, indexed in Pubmed: 33932268.

4. McMurray JJV, Packer M. How should we sequence the treatments for heart failure and a reduced ejection fraction? A redefinition of evidence-based medicine. Circulation. 2021; 143(9): 875-877, doi: 10.1161/CIRCULATIONAHA.120.052926, indexed in Pubmed: 33378214.

5. Velazquez EJ, Morrow DA, DeVore AD, et al. Angiotensin-Neprilysin inhibition in acute decompensated heart failure. N Engl J Med. 2019; 380(6): 539-548, doi: 10.1056/NEJMoa1812851, indexed in Pubmed: 30415601.

6. Wachter R, Senni M, Belohlavek J, et al. Initiation of sacubitril/valsartan in haemodynamically stabilised heart failure patients in hospital or early after discharge: primary results of the randomised TRANSITION study. Eur J Heart Fail. 2019; 21(8): 998-1007, doi: 10.1002/ejhf.1498, indexed in Pubmed: 31134724.

7. Morrow D, Velazquez E, DeVore A, et al. Clinical Outcomes in Patients With Acute Decompensated Heart Failure Randomly Assigned to Sacubitril/Valsartan or Enalapril in the PIONEER-HF Trial. Circulation. 2019; 139(19): 2285-2288, doi: 10.1161/circulationaha.118.039331, indexed in Pubmed: 30955360.

8. Glikson M, Nielsen JC, Kronborg MB, et al. 2021 ESC Guidelines on cardiac pacing and cardiac resynchronization therapy. Eur Heart J. 2021; 42(35): 3427-3520, doi: 10.1093/eurheartj/ehab364, indexed in Pubmed: 34455430 .

9. Anker SD, Butler J, Filippatos G, et al. EMPEROR-Preserved Trial Investigators. Empagliflozin in heart failure with a preserved ejection fraction. N Engl J Med. 2021; 385(16): 1451-1461, doi: 10.1056/NEJMoa2107038, indexed in Pubmed: 34449189.

10. Lelonek M, Stopczyńska I, Korościk E, et al. Multicenter experiences with levosimendan therapy and its safety in patients with decompensated advanced heart failure. Adv Clin Exp Med. 2020; 29(11): 1305-1312, doi: 10.17219/acem/126301, indexed in Pubmed: 33269816.

11. Grayburn PA, Sannino A, Packer M. Distinguishing proportionate and disproportionate subtypes in functional mitral regurgitation and left ventricular systolic dysfunction. JACC Cardiovasc Imaging. 2021; 14(4): 726-729, doi: 10.1016/j.jcmg.2020.05.043, indexed in Pubmed: 32861653.

12. Hawkins NM, Virani S, Ceconi C. Heart failure and chronic obstructive pulmonary disease: the challenges facing physicians and health services. Eur Heart J. 2013; 34(36): 2795-2803, doi: 10.1093/eurheartj/eht192, indexed in Pubmed: 23832490.

13. Verdecchia $P, C$ avallini $C$, Coiro $S$, et al. Certainties fading away: $\beta$-blockers do not worsen chronic obstructive pulmonary disease. Eur Heart J Suppl. 2021; 23(Suppl E): E172-E176, doi: 10.1093/eurheartj/suab116, indexed in Pubmed: 34650380.

14. Ponikowski P, Kirwan BA, Anker SD, et al. Ferric carboxymaltose for iron deficiency at discharge after acute heart failure: a multicentre, double-blind, randomised, controlled trial. Lancet. 2020; 396(10266): 1895-1904, doi: 10.1016/S0140-6736(20)32339-4, indexed in Pubmed: 33197395.

15. Elliott PM, Anastasakis A, Asimaki A, et al. Definition and treatment of arrhythmogenic cardiomyopathy: an updated expert panel report. Eur J Heart Fail. 2019; 21(8): 955-964, doi: 10.1002/ejhf.1534, indexed in Pubmed: 31210398.

16. Dziewięcka $E$, Rubiś $P$. Mortality risk assessment in dilated cardiomyopathy: the Krakow DCM Risk Score. Kardiol Pol. 2021; 79(2): 215-216, doi: 10.33963/KP.15827, indexed in Pubmed: 33635032.

17. Halliday BP, Wassall $R$, Lota AS, et al. Withdrawal of pharmacological treatment for heart failure in patients with recovered dilated cardiomyopathy (TRED-HF): an open-label, pilot, randomised trial. Lancet. 2019; 393(10166): 61-73, doi: 10.1016/S0140-6736(18)32484-X, indexed in Pubmed: 30429050.

18. Garcia-Pavia P, Rapezzi C, Adler $Y$, et al. Diagnosis and treatment of cardiac amyloidosis. A position statement of the European Society of Cardiology Working Group on Myocardial and Pericardial Diseases. Eur J Heart Fail. 2021; 23(4): 512-526, doi: 10.1002/ejhf.2140, indexed in Pubmed: 33826207

19. Maurer M, Schwartz J, Gundapaneni B, et al. Tafamidis Treatment for Patients with Transthyretin Amyloid Cardiomyopathy. N Engl J Med. 2018; 379(11): 1007-1016, doi: 10.1056/nejmoa1805689. 